\title{
Analysis of Sedimentary Cyclicity Based on X-ray CT Images and Ultrasonic Measurements of Core Samples
}

\author{
M.H. Grochau* (Petrobras), E. Campos (Petrobras), T.M. Mueller (CSIRO), \\ D. Nadri (CSIRO), B. Clennell (CSIRO) \& B. Gurevich (Curtin University of \\ Technology)
}

\section{SUMMARY}

We present a methodology to assess rock properties by using X-ray computed tomography (CT) images along with the ultrasonic $\mathrm{P}$-wave velocity measurements in laboratory under different confining pressures, and borehole logs from a stratigraphic interval in Campos Basin, offshore Brazil. The higher resolution of sampling in the core data overcomes the aliasing and smoothing effect of log-based measurements and enables the delineation of stratigraphic cycles. In addition, we observe a good correlation between X-ray $\mathrm{CT}$ value and ultrasonic P-wave velocity. Large variation in measured ultrasonic $\mathrm{P}$-wave velocity between different samples is related to variations in calcium content. This improved understanding of the cyclic nature of physical property variations may help to correlate stratigraphy between wells and provide valuable information for paleo-climate studies. This study also helps to understand the facies heterogeneity in fine scale which is needed for choosing the correct rock physics model for further undergoing studies such as time lapse in this field. Given the periodic nature of sedimentary facies, the unique combination of $\mathrm{X}$-ray CT images, ultrasonic $\mathrm{P}$-wave velocities, and high resolution well logs in this field could be used as an example for seismic wave propagation studies in periodic media. 


\section{Introduction}

Small-scale changes in rock properties, such as those resulting from centimetre scale depositional layering, are usually undetectable in standard borehole logs (Murphy et al., 1984). Even high resolution logs with a very small sampling interval (e.g. 2 inches), may still have relatively large investigation volume. This presents a problem when we wish to capture the full variation in physical properties for purposes such as rock physics modelling.

The environmental conditions during the sedimentation could cause periodic variations of carbonate calcium concentration of the sedimentary environment, reflecting periods of higher and lower ratios between pelagic carbonate plankton productivity and terrigeneous (clay, silt) sediment input. Burial diagenesis can enhance the original contrast between the compositionally distinct layers.

One method that gives useful information in an automated way and at a higher resolution than downhole logs is X-ray computed tomography (CT) of cores (Mees et. al., 2003). Marine sediment cores samples are the fundamental source of information on seabed character, depositional history and environmental change (Rothwell and Rack, 2006). The CT number encodes a combination of mass density and electron density proportional to mean atomic number.

In this paper we take advantage of a unique densely sampled CT and ultrasonic dataset in a deep shale environment offshore Brazil to demonstrate that these data provide valuable information on centimetre-scale heterogeneity that can be attributed to sedimentary cyclicity.

\section{Data}

The data come from a well in Campos Basin, offshore Brazil. The interval is approximately $2800 \mathrm{~m}$ below sea floor, in an Oligocene to Miocene hemipelagic sequence of alternating marls and shales that forms the overburden to the main sandstone reservoir. The data consist of CT images of the whole core, ultrasonic P-wave measurements on core plugs and well logs.

X-ray CT images were taken every $5 \mathrm{~cm}$ from cores at the depth of around $2800 \mathrm{~m}$. To acquire these CT images, we used a Picker - PQS scanner with X-ray voltage set at $130 \mathrm{kV}$ and current at $100 \mathrm{~mA}$. The time per scan was $4 \mathrm{~s}$ and image resolution $0.5 \mathrm{~mm}$ x $0.5 \mathrm{~mm}$. The standard deviation of the Xray CT value measurements is $0.56 \%$. The slice thickness is $1 \mathrm{~cm}$, meaning that the support volume of the measurements is smaller than the sampling interval. This is an important difference from the high resolution downhole density log, where the sampling interval is similar (2 inches $=5.08 \mathrm{~cm}$ ), but the support volume is larger $(10 \mathrm{~cm})$.

Ultrasonic (500 kHz) $P$-wave velocities were measured at different pressures on 23 rock samples, providing complementary information about their elastic properties. The measurements were done for horizontally and vertically oriented samples using a varying confining pressure from 1000 to 6000psi.

An extensive suite of borehole logs is also available, which includes high-resolution gamma ray and density logs with sample interval of $5.08 \mathrm{~cm}$. The sonic $P$ and $S$ velocities were measured by a monopole sonic logging tool with a spacing of $20 \mathrm{~cm}$ (standard) and $5.08 \mathrm{~cm}$ (high resolution).

\section{Methodology}

We initially investigate standard borehole logs over the interval to assess the rock physical properties. The resolution is not enough to detect any lithology intercalation in the rocks immediately above the sandstone reservoir. However, ultrasonic velocity measurements from different depths at different confining pressure show a substantial variation in elastic properties. High resolution logs show some indications of a cyclic pattern. These logs have a small sampling rate $(5.08 \mathrm{~cm})$; however the actual resolution is lower because the volume of investigation of various sensors is 2-6 times larger than the frame spacing at which data were collected. 
In order to get more insight about the variation of elastic properties, we use the regularly spaced X-ray CT scans performed on the whole core. We compute the mean X-ray CT value for each image by averaging it over a large circular area chosen to remove the outer part of the slices, to reduce the impact of beam hardening (Desrues et al., 2006). Figure 1a shows X-ray CT value in Hounsfield units (HU) for different depths. Figure $1 \mathrm{~b}$ shows CT values obtained from the HU values by conversion to an 8-bit grayscale. In the depth interval from $2862 \mathrm{~m}$ to $2870 \mathrm{~m}$ there is a clear signature of cyclicity. In the sandstone interval (2870m to $2874.3 \mathrm{~m}$ ), the X-ray CT values are lower and the cyclicity disappears.

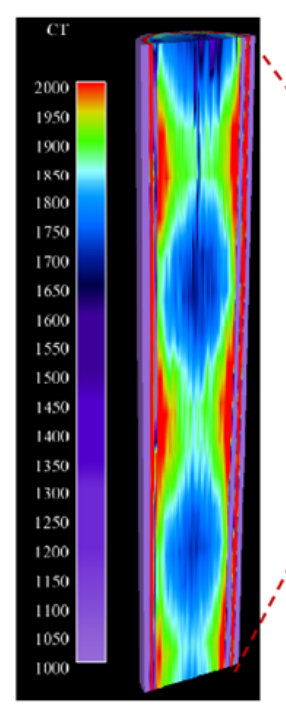

Hounsfield Units (HU)

(a)
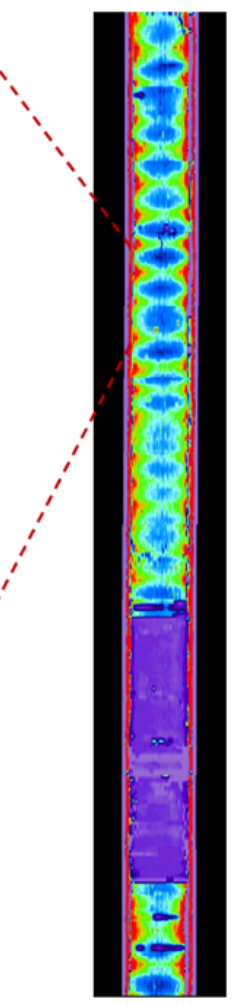

2862

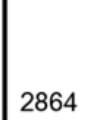

2864

2866

2868

2870

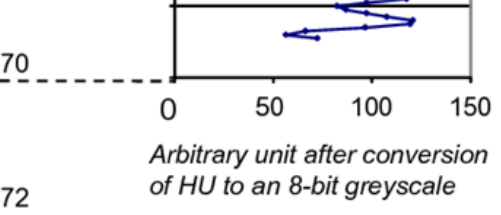

2872

of $\mathrm{HU}$ to an 8-bit greyscale

(b)

Figure $1 X$-ray $C T$ values obtained by computing the average value over a circular area for each of the 143 CT images at every $5 \mathrm{~cm}$. Alternating low and high CT values provide evidence of cyclicity. Hounsfield units (a) and arbitrary unit after conversion to an 8-bit greyscale (b).

2874

Using correlation between mass density measured on core samples and X-ray CT value, we compute so-called X-ray densities (or "mean density from CT"). Next, we crossplot ultrasonic $P$ wave velocity versus mean X-ray density of the corresponding samples (Figure 2). We select velocities corresponding to the estimated in-situ confining pressure (3000 psi), by taking into account lithostatic stress. Figure 2 shows good correlation between $\mathrm{X}$-ray density and $P$-velocity. This regression line is then used to compute the velocities as a function of density for each CT image. Figure 2 also shows CT images from shale and marlstone samples. Shales are related to dark images with smaller densities and velocities; marlstones are in the other extreme, and in between there is a gradation between the two. These changes are caused by the differing amount of calcium carbonate, which is enhanced in CT imagery owing to the relatively high atomic number of calcium.

\section{Results}

The computed velocities from CT images show clearly the cyclic behaviour with much higher resolution than the sonic log (Figure 3). However, comparison between these velocities and the sonic log shows discrepancy in the upper part of the interval. Detail studies from core samples did not confirm the presence of seismic anisotropy. Dispersion might cause small variations (lab measurements were done on partially dried samples) but not at this magnitude. We believe that the small scale heterogeneity could cause the misfit between sonic log and ultrasonic measurements. 
Figure 2 Ultrasonic $P$-wave velocity measured from plugs versus relative $X$-ray density from CT images, obtained at the same position. The correlation coefficient is 0.56. Red and blue symbols correspond to Vp values for vertically and horizontally oriented samples, respectively. CT images from samples $3 \quad(2863.25 \mathrm{~m})$ and 8 (2864.40m) are also shown. Bright colours mean higher $X$-ray $C T$ values (higher mass densities, higher P-wave velocities), whereas dark colours correspond to low CT values.

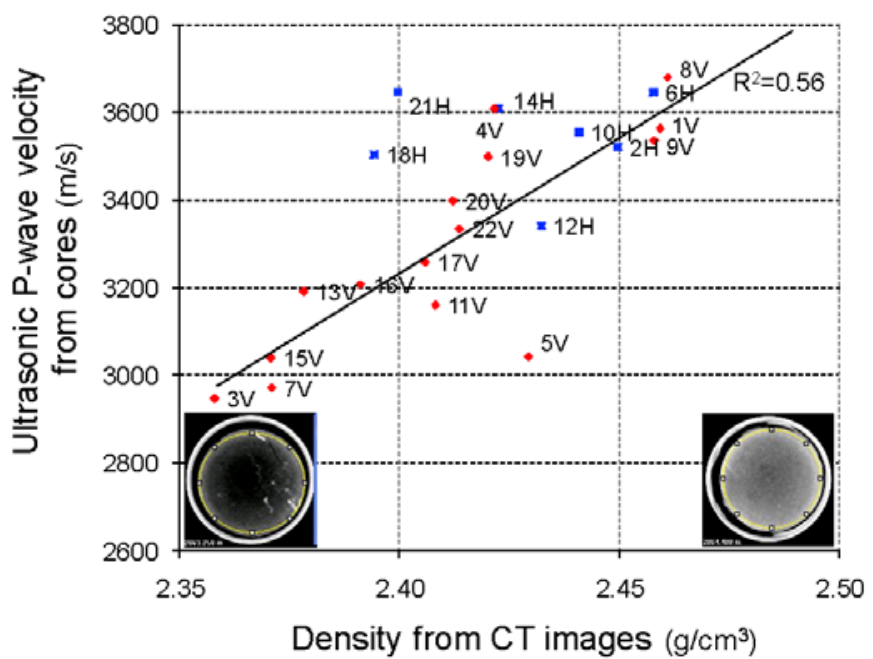

Vertical variation of X-ray CT values shows a clear pattern of sedimentary cyclicity with a spatial period of $37.7 \mathrm{~cm}$ estimated from the autocorrelation function (Figure 4) and the periodogram.

Besides providing the elastic properties, CT images also facilitate the analysis of ultrasonic P-wave velocity of core samples by discriminating different lithofacies. Figure 5 shows the ultrasonic P-wave velocity at different confining pressures for 23 samples. Samples with high concentration of calcium carbonates (magenta) have higher velocity than the shaley samples (green). Intermediate concentrations of clay and carbonate calcium show velocities in between (yellow). Without imposing the lithology constraint it would be difficult to make inference about the acoustic properties of the core samples and choosing a right rock physics model for further studies, in particular for time lapse feasibility analysis that will be performed in this field.

Figure 3 Ultrasonic $P$-wave velocity from plugs at reservoir effective pressure (3000psi) measured on vertically (red symbols) and horizontally (blue symbols) oriented core plugs; P-wave velocity from high resolution sonic log (blue line) and velocity derived from CT images (black line). Magenta, yellow and green colours are related to higher (contains more CaCO3), medium and lower (contains more shale) velocities computed from CT images.
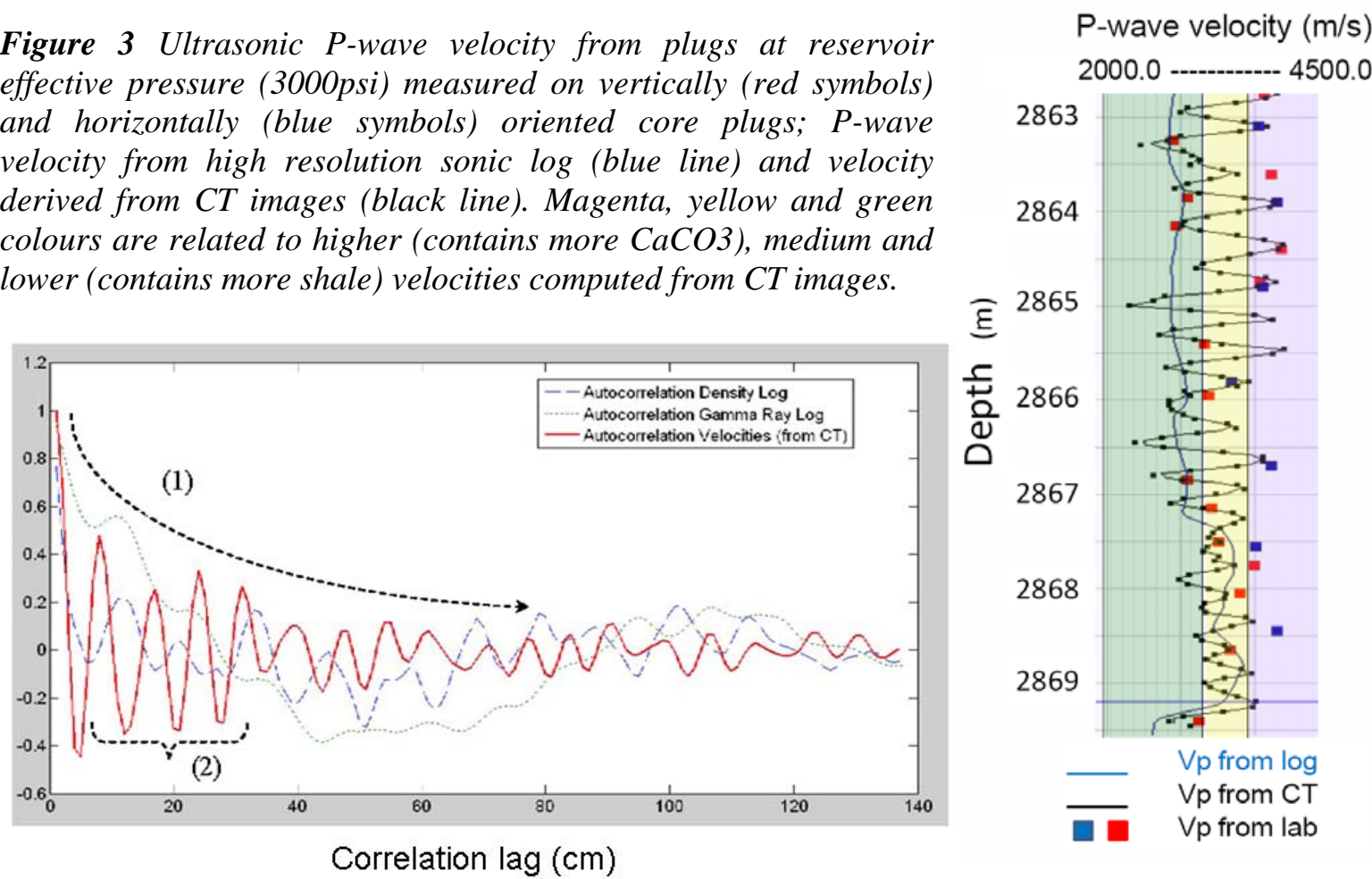

Figure 4 (a) Autocorrelation function of gamma ray log (green dots), density log (blue dashed line) and CT velocities (red line) for the depth interval shown in Figure 3. The autocorrelation function of the CT velocities consists of two parts: a signature of a random process showing a decrease of correlation function with correlation lag (1) and a signature of a periodic (cyclic) process with a period of $37.7 \mathrm{~cm}(2)$. 


\section{Discussion and conclusions}

$\mathrm{X}$-ray density obtained from CT images shows a good correlation with ultrasonic $P$-wave velocity. This correlation is better than X-ray density with mass density due to that fact that variations in calcium carbonate content have a strong effect on both velocity and X-ray CT, but lesser effect on mass density. Higher content of calcium carbonate causes stiffening and shifts the $V p$ curve to higher velocities.

We have shown that densely sampled X-ray CT images over a shale/marl interval reveal small scale sedimentary heterogeneity and aid in the interpretation of petrophysical and rock physics measurements. In particular, we demonstrate that there is spatial cyclicity that cannot be revealed even using high resolution logs. A good match between measured ultrasonic velocities and those computed from CT shows that the large variation of ultrasonic velocities between different samples is caused by vertical heterogeneity (variation of calcium carbonate content) and not by strong anisotropy. The cyclic variations in X-ray CT values disappear completely in the sand reservoir (turbidite). The cyclicity also provides valuable information for paleo-climate and time lapse studies.

Figure 5 Ultrasonic $P$-wave velocities (23 plugs) versus confining pressure measured in the laboratory. Core plugs have been classified based on CT X-ray absorption for further rock physics based analysis. Magenta, yellow and green colours are related to higher, middle and smaller ranges of velocity.

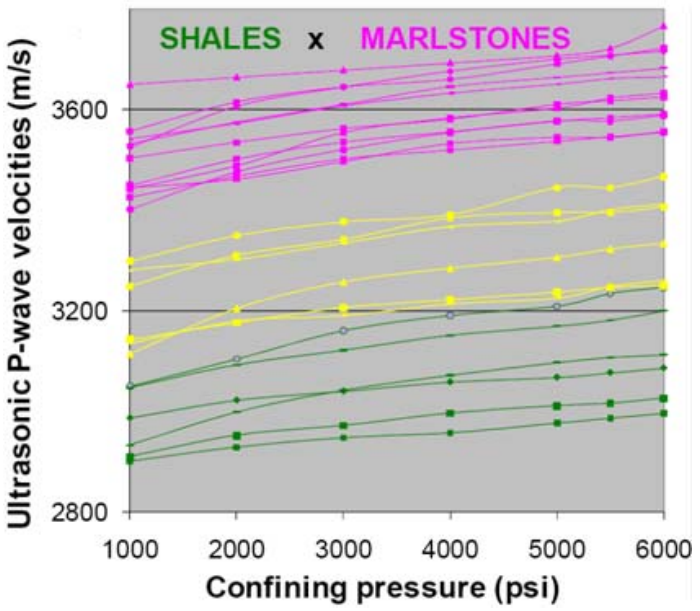

\section{Acknowledgments}

We acknowledge Petrobras for the provision of log and core data, and permission to publish this paper. We would also like to thanks the following people for their ideas, opinions and comments: Ana Moliterno, Armando Scarparo Cunha, Gilberto Albertao, Guilherme Vasquez and Osni de Paula of Petrobras; Maxim Lebedev of Curtin University of Technology.

\section{References}

Desrues, J., G. Viggiani and P. Besuelle, 2006. Advances in X-ray tomography for geomaterials, International Workshop on X-ray CT for Geomaterials, Aussois, France, ISTE.

Mees, F., R. Swennen, M. Van Geet, and P. Jacobs, 2003. Applications of X-ray computed tomography in the geosciences, Geological Society, London, Special Publications, 215, 1-6.

Murphy, W. F. I., J. N. Roberts, D. Yale, and K. W. Winkler, 1984. Centimeter scale heterogeneities and microstratification in sedimentary rocks, Geophysical Research Letters, 11, 697-700.

Rothwell, R.G. and F. R. Rack, 2006. New techniques in sediment core analysis: an introduction, Geological Society London, Special Publications, 267, 1-29. 